\title{
Representação e literacia dos dados: o caso das empresas de design em Portugal
}

\author{
$10^{\text {th }}$ Information Design International Conference (CIDI 2021)
}

Nina Costa, Rui Costa, Vasco Branco, Afonso Borges, Raul Cunca, Ana Catarina Silva, António Modesto

design de informação, literacia dos dados, educação em design, empresas de design em Portugal

\begin{abstract}
Este estudo resulta de um exercício desenvolvido dentro do âmbito do projeto nacional "Para um observatório de design em Portugal: modelos, instrumentos, representações, estratégias", com 15 alunos de mestrado da Universidade de Aveiro. Objetivou mapear dados económicos e financeiros sobre 1200 empresas de design em Portugal e capacitar jovens designers na interpretação e representação de dados. Durante um semestre, os alunos categorizaram, curaram, densificaram e mapearam a informação sobre essas empresas. Usaram essa informação para desenvolver uma infografia, com abstrações e metáforas, constituindo a sua primeira experiência de manuseamento e comunicação de dados. Os resultados preliminares indicam uma emancipação importante por parte dos alunos, que demonstraram interesse e capacidade crítica, na interpretação dos dados. Este estudo constitui mais um passo rumo à consolidação de uma abordagem participativa e distribuída de observação, essencial para a construção de um observatório de design em Portugal.
\end{abstract}

information design, data literacy, design education, design companies in Portugal

This study is the result of an exercise developed within the scope of the project "Towards a design observatory in Portugal: models, instruments, representations, strategies", with 15 master students from the University of Aveiro. It aimed to map economic and financial data of 1200 design companies in Portugal and to train novice design students to start their journey in data interpretation and representation. During the period of one semester, students categorized, cured, densified and mapped information about these companies. They used this information to develop infographics, with abstractions and metaphors, constituting their first experience with data manipulation and representation. The preliminary results indicate an important emancipation for students, who showed interest and critical capacity to interpret the data. This study is another step towards the consolidation of a participatory and distributed observation approach, essential for the construction of a design observatory in Portugal.

\section{Observar o design em Portugal}

Desde 2013, com a extinção do Centro Português de Design (Curado, 2013), não existe um instrumento de política pública que sirva de interface entre o ecossistema de design nacional e o tecido socioeconómico Português. Pela falta de visibilidade, o design torna-se cada vez mais operativo, enquanto fator de inovação e desenvolvimento. Um inquérito distribuído a nível Europeu para avaliar a inovação no continente revela por exemplo que, em Portugal, apenas

Anais do $10^{\circ} \mathrm{CIDI}$ e $10^{\circ} \mathrm{CONGIC}$

Kelli C.A.S. Smythe, Rafael de Castro Andrade (orgs.)

Sociedade Brasileira de Design da Informação - SBDI

Curitiba | Brasil | 2021
Proceedings of the $10^{\text {th }} \mathrm{CIDI}$ and $10^{\text {th }}$ CONGIC

Kelli C.A.S. Smythe, Rafael de Castro Andrade (orgs.)

Sociedade Brasileira de Design da Informação - SBDI Curitiba | Brazil | 2021 
$13 \%$ das empresas afirmam integrar design a nível estratégico, enquanto $49 \%$ não o integram sistematicamente (Innobarometer, 2016).

Nesse sentido, o projeto DesignOBS, "para um Observatório de design em Portugal: modelos, instrumentos, representações e estratégias", tem como objetivo a recolha, sistematização e o mapeamento de informação e dados existentes sobre a disciplina no país, olhando para os diferentes vetores do ecossistema (ex. atores, utilizadores, designers etc.), adaptando modelos de existentes replicados na Europa (Whicher et al., 2016) usados para apoiar o desenvolvimento de melhores políticas públicas para o design. A estratégia de observação e representação é, no entanto, diferente das demais. Tendo em conta a falta de infraestrutura dedicada à investigação e promoção da disciplina (Costa et al., 2021c), a adoção de uma abordagem mais distribuída e participativa foi desenvolvida e aplicada, numa primeira fase, no vetor da investigação do design em Portugal (Costa et al., 2020a; 2021b). Este primeiro estudo de caso, objetivou compreender a quantidade e qualidade dos doutoramentos de design realizados no país e mapear as suas conexões. Recolheram-se todas as teses desenvolvidas na área através das bases de dados governamentais e apelou-se à rede de escolas de design nacionais (Borges et al.,2017) para ajudar nessa recolha e leitura. A sistematização e curadoria da base de dados, atualmente disponível para leitura pública no website do projeto (Costa et al. 2020b) permitiu realizar uma análise contínua de dados e criou o espaço para a emergência de novos questionamentos. Além de compreender, empiricamente, que a área de investigação doutoral em design é povoada por inúmeras contribuições por vezes, mais focadas nas artes, uma análise e representação em rede focadas nas teses de doutoramento mencionadas nesse conjunto de registos, revelou a falta de conexão e "ilhas de pensamento" existente no conjunto que caracteriza a investigação doutoral na área em Portugal (Costa et al., 2021b). Este exercício cíclico de recolha, análise, mapeamento e interpretação; e emergência de novo(s) questionamento(s), é um processo intrínseco à representação no âmbito do design de informação (Costa, R. 2014) e é algo que, enquanto equipa, procuramos enfatizar neste processo de cocriação do observatório de design em Portugal. No entanto, encontramos desafios importantes, sobretudo no fecho desses ciclos. Neste breve resumo, enfatizamos um deles, nomeadamente, no convite e promoção de uma participação mais ativa dos designers na leitura da sua própria disciplina.

\section{Recolha, interpretação e representação de dados}

Com a disponibilização massiva de dados, a nível mundial, além da sua produção é cada vez mais importante compreender como os usar e comunicar. No entanto, ainda se verifica um profundo desequilíbrio entre os que os produzem (ex. cidadãos) e os que têm capacidade e competência para os usar de forma (ex. especialistas, empresas) (D'Ignasio, 2017). Os dados são um recurso, uma matéria prima cujo valor é apenas proporcional à capacidade de diversos atores (sejam empresas, organizações, ou pessoas individuais) de os lerem, transformá-los em algo passível de compreensão para incentivar, gerar mudança e/ou inovar. De acordo com Kapoor et al. (2015), um sistema de inovação é caracterizado por iterações entre sistemas de 
registo (ex. dados), sistemas de conhecimento (ex. questões, reflexões, ferramentas, instrumentos) e sistemas de envolvimento (ex. colaborações, interações). Iniciativas tais como a Open Government Initiative, nos Estados Unidos (ver Kapoor et al., 2017) lançaram múltiplas bases de dados, sobre vários tópicos relacionados com os cidadãos das cidades (ex. uso da energia) que foram basilares para que várias empresas/instituições, com recurso a especialistas, pudessem gerar novos produtos e serviços com base em informação real. Mais recentemente, o Open4Citizens - projeto que envolveu uma rede de cinco instituições europeias - disponibilizou um toolkit para apoiar o uso de dados através da organização de hackatons participativas, visando sensibilizar a população geral para a importância dos dados, e assim desenvolver soluções para problemas sociais em conjunto com cidadãos, IT experts, administradores públicos, startups, e/ou outros atores (Concilio et al., 2017; Morelli et al., 2018, 2019). Após a conclusão desse projeto, abriram uma associação - NOODL.EU, The European Network of Open Data Labs - cujo intuito é o de multiplicar a iniciativa noutros países e regiões. Os efeitos a longo prazo deste tipo de projetos são, no entanto, ainda desconhecidos.

$\mathrm{Na}$ área do design especificamente, o trabalho desenvolvido pelo Politécnico de Milão, Itália; e a Universidade de Aalborg, na Dinamarca, também são cada vez mais importantes para explorar o papel dos designers nesta área. Mauri e a sua equipa (Mauri et al., 2918; 2021; Mauri, 2020) trabalham desde 2004 num programa que se foca na transformação de dados em representações visuais e digitais. Focam-se na análise e representação de desafios societais europeus (ex. emigração, ambiente) para envolver estudantes de mestrado em design na jornada de recolha, exploração e representação de dados com significado. Esse programa baseado em estúdio resulta em trabalhos de qualidade, expostos em websites, ou fisicamente no Campus de Milão sendo, no entanto, focado num público com um forte background na área de design gráfico/comunicação.

Por outro lado, De Götzen et al. (2018), exemplifica como a infusão da literacia dos dados em cursos de design de serviços pode beneficiar equipas no desenvolvimento de novas propostas de valor. Explora, com alunos de múltiplos backgrounds, sem experiência na manipulação de dados, como é que podem usar o conhecimento gerado e técnicas complementares na fase inicial do processo de design, para melhorar as suas soluções.

Estas abordagens são muito importantes para a formação de jovens designers, incentivando-os a usar esta matéria prima para melhorar seu processo e soluções. No entanto, verifica-se que os designers "ainda entendem que a interpretação de dados é algo exterior à sua disciplina" (Mauri, 2020).

\section{Metodologia}

Com base nos estudos/insights anteriores e objetivos do projeto DesignOBS, o desafio foi lançado a uma turma de 15 alunos de mestrado em Design da Universidade de Aveiro, no sentido de criar a sua própria análise e interpretação de um conjunto de dados focados na performance económico-financeira de 1200 empresas de design (código de atividades 74.10) 
em Portugal. Estes dados foram recolhidos pela equipa do DesignOBS, através da $S A B I$, Bureau Van Djik, um repositório com informação sobre mais de 800.000 empresas portuguesas. A base de dados continha informação sobre marca, localização (endereço, condado, distritos e Nuts II), website (quando disponível), número de telefone (quando disponível), volume de negócios (€), margem de lucro (\%), número de funcionários, custos com funcionários, resultados operacionais, lucros operacionais e a forma jurídica das empresas. Ao longo de um semestre letivo, com aulas predominantemente online devido ao contexto pandémico e múltiplas reuniões, os alunos criaram cinco outputs (infografia/poster), com relatórios, seguindo um processo de três fases:

1. sensibilização e preparação (introdução aos conceitos básicos de visualização e experimentação de representação livre, em médium físico ou digital e introdução ao Excel e RawGraph);

2. exploração e densificação de dados (categorização de dados, adição de informação)

3. representação e teste (uso de elementos multimédia para desenvolver infografia e apresentação final).

Além do acompanhamento semanal, recolha de relatórios intermédios e trabalhos finais, foram efetuadas entrevistas com os alunos para compreender o processo transformativo e potencial emancipador desta abordagem, aplicada num contexto de aprendizagem, com designers principiantes, cujo objeto/matéria prima são dados focados num dos vetores deste ecossistema: as empresas de design e/ou designers per si.

\section{Resultados preliminares}

Além da densificação da base de dados com novas informações (ex. categorização da área de atuação da empresa, tal como design industrial, comunicação, moda etc.), os alunos demonstraram capacidade crítica na curadoria dos dados apresentados, criando cinco posters com múltiplas informações que representam parte ou a totalidade das empresas presentes na base de dados fornecida. Esses posters, atualmente disponíveis na plataforma DesignOBS (www.designobs.pt), contêm um conjunto de metáforas e abstrações que transmitem uma mensagem em relação a esta indústria: no "Fish Design" figuram peixes de diferentes dimensões e formas que fazem alusão à constituição da indústria do design português como um ecossistema marinho, sendo a maioria microempresas (sardinhas). "À conquista do design" enfatiza a existência de "cavalos de Tróia" na arena - empresas com código de atividades de design (74.10) mas que, após análise de produtos e serviços, não se enquadram nessa atividade (ex. empresas de reprografia). "Exportação sobre rodas" usa a disposição geográfica do território para representar as exportações por região evidenciando a importância dos grandes centros urbanos para a área. "Limonada de design" usa a analogia do termo para demonstrar que, empresas com bom volume de negócio não geram necessariamente mais lucros. Finalmente, o "Panorama do design em Portugal" usa contentores como analogia às 
percentagens de exportações por setor, e o lixo no oceano alusivo às percentagens de prejuízo dessas empresas.

A análise de dados também demonstra que a transformação dos estudantes ao longo deste processo foi notória. Receio era o seu principal sentimento no início do processo, apresentando dificuldades em compreender o excel e muito menos como é que iriam gerar representações a partir de conjunto de dados tão extenso num poster/infografia. $\mathrm{O}$ "clique" (como um aluno mencionou) deu-se quando perceberam como é as perguntas os poderiam libertar da informação quantitativa na base de dados. Isto é, exploraram como é que a abstração, e a relação entre símbolos, cores e formas poderiam ser vetores de uma mensagem comunicada através de um medium, de forma criativa, criando uma ponte entre os dados, o designer, a mensagem e o leitor. A utilização de uma perspetiva crítica e criativa permitiu uma transformação interna: "mudei a perspetiva em relação aos dados (...)"; "aquele excel... dá para explorar muita coisa ainda e ver coisas de que se calhar não tínhamos noção" (testemunho de dois alunos). Esta transformação, primeiro interna, é materializada através da ação do designer - a criação de um artefato enfatiza uma mensagem sobre a qual o leitor/intérprete pode agir e refletir ("acho muito interessante, o facto de pegar num excel tão grande e transformá-lo numa coisa bonita, agradável para outras pessoas compreenderem e explorarem"). Esta mediação cultural entre os dados, e a missão do designer, da mediação cultural, é portanto, evidenciada e enfatizada neste exercício.

\section{Conclusões}

Este estudo, ainda em desenvolvimento, constitui um passo adicional na cocriação de um processo participativo e distribuído, alicerce essencial para o observatório de design em Portugal. A abertura das bases de dados contendo informação sobre o ecossistema de design, não é per se suficiente para inovar de forma sistemática. Este exercício, realizado com estudantes de design de várias áreas (industrial, comunicação, artes etc.) resultou não só num conjunto de infografias, mas também numa transformação na percepção do seu potencial papel no mundo dos dados, na capacitação dos designers enquanto intérpretes do ecossistema da sua própria disciplina, tarefa que, até à data, parece ser maioritariamente desempenhada por outras disciplinas, dedicadas às ciências sociais, gestão ou outras, mais analíticas. A expansão deste estudo a outras escolas e partes do território é essencial para o desenvolvimento de novas práticas, para desenvolver, através de dados, novos artefactos mediadores de mensagens.

\section{Agradecimentos}

Este artigo é resultado do projeto Design Obs. Para um Observatório do Design em Portugal: modelos, instrumentos, representação e estratégias, apoiado pelo Programa Operacional Regional de Lisboa (LISBOA 2020) e pelo Programa Operacional de Competitividade e Internacionalização (POCl-01-0145-FEDER-032445), no âmbito do Partnership Agreement 
PORTUGAL 2020, através do Fundo Europeu de Desenvolvimento Regional (FEDER) e da FCT - Fundação para a Ciência e Tecnologia.

\section{Referências}

Borges, A., Silva, A. C., Modesto, A., Cunca, R., Costa, R. C., \& Branco, V. (2018). REDE\#01: Reunião de Escolas de Design. Retrieved from http://hdl.handle.net/10773/24199

Concilio, G., Molinari, F., \& Morelli, N. (2017). Empowering citizens with open data by urban Hackathons. Proceedings of the 7th International Conference for E-Democracy and Open Government, CeDEM 2017, (March 2021), 125-134.

https://doi.org/10.1109/CeDEM.2017.28

Costa, N., Branco, V., Costa, R., Borges, A., Modesto, A., Silva, C., \& Cunca, R. (2020a). Towards a Design Observatory: the Case of Scholarly Design Research in Portugal. Proceedings of the Design Society: DESIGN Conference, 1, 827-836. https://doi.org/10.1017/dsd.2020.327

Costa, N., Branco, V., Costa, R., Borges, A., Modesto, A., Silva, C., \& Cunca, R. (2020b). Design doctorates undertaken in Portugal (Version 2) [Data set]. Zenodo. http://doi.org/10.5281/zenodo.4559415

Costa, N.; Branco, V.; Costa, R.; Borges, A.; Modesto, A.; Cunca, R.; Silva, A., DesignOBS (2021a): crafting a distributed design observation approach, CUMULUS'21, 8-11 June, Roma, Italy, http://hdl.handle.net/10773/30629

Costa, N.; Branco, V.; Costa, R.; Borges, A.; Modesto, A.; Cunca, R.; Silva, A. (2021b), Mapping the Research thread of PhDs in Design: a PhD citation analysis of the Portuguese doctorates, 8th International Conference on Research into Design, 7-10 January 2021, IDC School of Design, IIT, Bombay, India, http://hdl.handle.net/10773/30627

Costa, N.; Branco, V.; Costa, R.; Borges, A.; Modesto, A.; Cunca, R.; Silva, A. (2021c), Design Infrastructures: proposing alternative strategies for countries with a lower maturation of designculture, 12th International Conference on Applied Human Factors and Ergonomics (AHFE 2021) and the Affiliated Conferences, New York, July 25-29, http://hdl.handle.net/10773/30631

Curado, R. (2013). Centro Português de Design vai ser extinto. 1-6. Retrieved from http://www.dn.pt/artes/interior/centro-portugues-de-design-vai-ser-extinto-3248523.html

D'Ignazio, C. (2017). Creative data literacy. Information Design Journal, 23(1), 6-18. https://doi.org/10.1075/idj.23.1.03dig

De Götzen, A., Simeone, L., Morelli, N., \& Kun, P. (2018). Making Sense of Data in a Service Design Education. ServDes 2018 Service Design Proof of Concept, (June). Retrieved from http://ualresearchonline.arts.ac.uk/7712/1/Mapping-and-Devloping-SDR-in-the-UK.pdf

European Commission. (2016). Innobarometer 2016 - EU business innovation trends.

Kapoor, S., Mojsilovi, A., Strattner, J. N., \& Varshney, K. R. (2015). From Open Data Ecosystems to Systems of Innovation: A Journey to Realize the Promise of Open Data. Bloomberg Data for Good Exchange Conference.

Mauri, M., Colombo, G., Briones, M. D. L. Á., \& Ciuccarelli, P. (2019). Teaching the critical role of designers in the data society: the DensityDesign approach. 
Mauri, M. (2020). Introducing Information Visualization to Design Students. EDULEARN20 Proceedings, 1(July), 4442-4448. https://doi.org/10.21125/edulearn.2020.1173

Mauri, M., Colombo, G., Briones, M., \& Ciuccarelli, P. (2021). Teaching the critical role of designers in the data society: the DensityDesign approach. https://doi.org/10.21606/learnxdesign.2019.12046

Morelli, N., de Götzen, A., \& Simeone, L. (2018). A system of innovation to activate practices on open data: The Open4Citizens project. Proceedings of 3rd International Conference on Smart Learning Ecosystem and Regional Development - The Interplay of Data, Technology, Place and People. Presented at the Aalborg, Denmark. Aalborg, Denmark.

Morelli, N., de Götzen, A., \& Simeone, L. (2019). A System of Innovation to Activate Practices on Open Data: The Open4Citizens Project. (May 2018), 99-109. https://doi.org/10.1007/978-3319-92022-1 9

Whicher, A. (2016). Benchmarking Design for Innovation Policy in Europe.

\section{Sobre os autores}

Nina Costa Dra., Universidade de Aveiro, Instituto de Design, Média e Cultura (ID+), Portugal ndc@ua.pt

Rui Costa Dr. UA, ID+, Portugal

Vasco Branco Dr. UA, ID+, Portugal

Afonso Borges Dr. UA, ID+, Portugal

Raul Cunca Dr. Faculdade de Belas Artes da Universidade de Lisboa, ID+, Portugal

Ana Catarina Silva, Instituto Politécnico de Cávado e do Ave, ID+, Portugal

António Modesto, Faculdade de Belas Artes da Universidade do Porto, ID+, Portugal 